\title{
Estudio comparativo de tres técnicas de hemodiálisis: AFB-K, Hemodiafiltración y Hemodiálisis de alto flujo
}

\author{
Premio de Investigación en Enfermería Nefrológica Janssen- Cilag
}

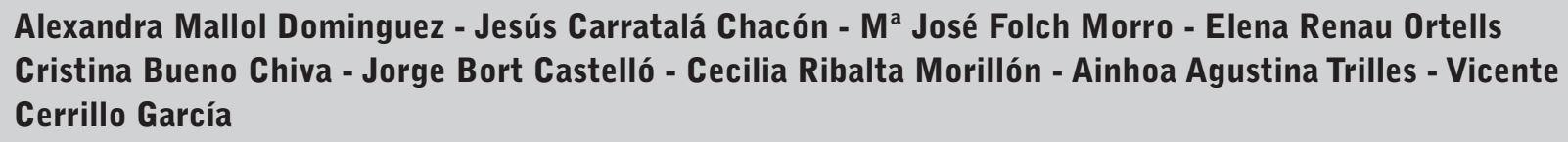

Alexandra Mallol Dominguez - Jesús Carratalá Chacón - Ma José Folch Morro - Elena Renau Ortells Cristina Bueno Chiva - Jorge Bort Castelló - Cecilia Ribalta Morillón - Ainhoa Agustina Trilles - Vicente Cerrillo García

Unidad de Diálisis. Hospital General de Castellón

\section{Resumen}

La investigación en nefrología pretende encontrar técnicas cada vez más eficientes de depuración extrarrenal. La técnica dialítica biofiltración sin acetato con perfil de potasio pretende ofrecer una mejor tolerancia y reducir el riego de arritmias. Nuestro objetivo fue comparar está técnica con la hemodiálisis de alto flujo y la hemodiafiltración con el fin de averiguar que tratamiento dialítico es más adecuado para cada paciente.

Estudio controlado, prospectivo y cruzado en el cual los mismos pacientes han sido su propio control. La elección de los pacientes y de la técnica fue aleatoria dializándose los pacientes durante 13 sesiones consecutivas con cada una de las técnicas objeto del estudio. Durante todo el estudio se mantuvieron constantes los siguientes parámetros de diálisis: duración de la sesión, flujo, calibre de las agujas y dosis de heparina. A los quince días de comenzar cada período se realizó a los pacientes un electrocardiograma y una analítica (todo pre y post diálisis).

En las técnicas biofiltración y hemodiafiltración el valor del bicarbonato es más fisiológico. Con la biofiltración y la hemodiálisis de alto flujo se consi-

\begin{tabular}{c} 
Correspondencia: \\
Alexandra Mallol Dominguez \\
Unidad de Diálisis. Hospital General de Castellón \\
Avda. Benicasim s/n \\
12004 Castellón \\
alexmallol@hotmail.com \\
\hline
\end{tabular}

gue una mejor corrección del equilibrio ácido-base. En cuanto a los valores de sodio existen pequeñas diferencias en las tres técnicas estando éstos en todo momento dentro del intervalo fisiológico. En la hemodiafiltración los episodios de arritmias aumentaron. Los tres métodos han demostrado una correcta eficacia dialítica, tolerancia y estabilidad cardiovascular.

PALABRAS CLAVE:

- EFICACIA DIALÍTICA

- BIOFILTRACIÓN SIN ACETATO CON PERFIL DE POTASIO (AFB-K)

- HEMODIAFILTRACIÓN

- HEMODIÁLISIS DE ALTO FLUJO

Comparative study of three haemodialysis techniques: AFB-K, Haemodiafiltration and High-flow Haemodialysis

\section{Abstract}

Research in nephrology seeks to find increasingly efficient extrarenal purification techniques. The dialysis technique of potassium-profiled acetatefree biofiltration offers better tolerance and reduces the risk of arrhythmias. Our aim was to compare this technique with high-flow haemodialysis and with haemodiafiltration in order to ascertain which dialysis treatment is the most suitable for each patient. 
A prospective, controlled cross study in which the same patients were their own control group. The choice of patients and technique was random, with the patients undergoing 13 consecutive sessions of dialysis with each of the techniques being studied. Throughout the study, the following dialysis parameters were kept constant: duration of the session, flow, calibre of the needs and dose of heparin. Fifteen days after starting each period, patients were given an electrocardiogram and blood test (all pre and post dialysis).

With the biofiltration and haemodiafiltration techniques the bicarbonate value is more physiological. With biofiltration and high-flow haemodialysis a better correction of the acid-base balance is achieved. As regards sodium value, there are small differences in the three techniques, always within the physiological interval. In haemodiafiltration, episodes of arrhythmia increased. The three methods have shown correct dialysis efficiency, tolerance and cardiovascular stability.

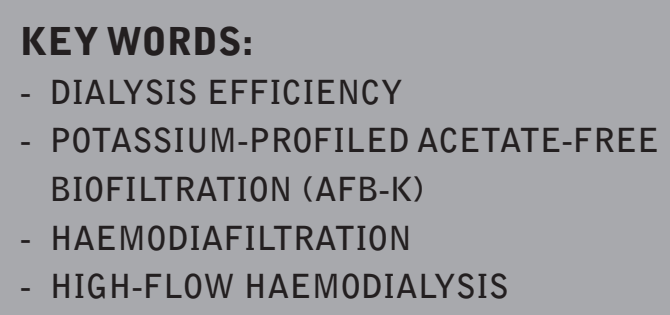

\section{Introducción}

La investigación en nefrología pretende constantemente encontrar formas más eficientes de depuración extrarrenal. Aunque la hemodiálisis convencional fue, en un principio, el tratamiento de elección de la enfermedad renal crónica no dejaba de plantear inconvenientes derivados de su duración, de la dep uración insuficiente y de la mala tolerancia de la técnica. La capacidad innovadora de la nefrología intenta continuamente superar estos obstáculos desarrollando técnicas que permitan hacer una depuración de la sangre más rápida, más eficaz y mejor tolerada por el paciente.

Recientemente se ha introducido en nuestra unidad una nueva técnica dialítica: la Biofiltración sin acetato con perfil de potasio (AFB-K), por ello hemos decidido elaborar un estudio comparándola con otras dos técnicas: Hemodiálisis de alto flujo (HD-AF) y Hemodiafiltración (HDF).

La HDF es una técnica dialítica que utiliza los mecanismos depurativos de difusión y convección simultáneamente ${ }^{1,2}$. La HD-AF: es similar a la HDF salvo que no utiliza líquido de reposición. Debido a ello disminuye la depuración de medianas moléculas y aumenta el riesgo de retrofiltración.

La AFB-K, es una técnica que no utiliza acetato ni bicarbonato en el líquido de diálisis, lo cual favorece la mejor tolerancia del tratamiento dialítico $0^{3,4}$ y además permite realizar un perfil descendente de concentración de potasio en el líquido de baño, facilitando una eliminación gradual del exceso de potasio durante la sesión de hemodiálisis, con lo cual se evita la caída brusca del mismo y se reduce el riesgo de aparición de arritmias 3,5,6. Esta técnica permite lograr el doble objetivo de eliminar las elevadas cantidades de potasio y mantener minuto a minuto un gradiente de concentración limitado entre la sangre del paciente y el líquido de diálisis ${ }^{5,6}$. Además, goza de las mismas ventajas que la HDF, pues también utiliza dializadores de alta permeabilidad y un determinado volumen de líquido de sustitución (9-12 litros) que, en este caso, está compuesto de bicarbonato sódico en una concentración de $166 \mathrm{mEq} / \mathrm{litro}$.

\section{Objetivo}

Nuestro objetivo fue comparar está técnica con la hemodiálisis de alto flujo y la hemodiafiltración, con el fin de averiguar que tratamiento dialítico es más adecuado para cada paciente.

\section{Material y Método}

Hemos realizado un estudio controlado, prospectivo y cruzado en el cual los mismos pacientes han sido su propio control. Para ello hemos seleccionado una muestra de 20 pacientes, 17 hombres y 3 mujeres con edad de $68,7 \pm 11$ años. De ellos, 13 eran portadores de FAVI, 2 de injerto protésico y 5 con catéter tunelizado. Su estancia en programa de diálisis era de $58,5 \pm 27,7$ meses. El peso seco que presentaban era de $69,9 \pm 15,5 \mathrm{Kg}$. Todos ellos se dializaban anterior- 
mente con HDF, con una perfusión total de 6-9 litros durante la sesión dialítica.

La duración del estudio de campo fue de 3 meses. Cada paciente se dializó durante 13 sesiones consecutivas con cada una de las tres técnicas. La elección de los pacientes y técnicas fue aleatoria. Las tres técnicas se realizaron simultáneamente de la siguiente manera:

- Primer período: 6 pacientes con AFB-K, 4 con HD-AF y 10 con HDF

- Segundo período: 8 pacientes con AFB-K, 8 con HD-AF y 4 con HDF

- Tercer período: 6 pacientes con AFB-K, 8 con HD-AF y 6 con HDF

Durante todo el estudio se mantuvieron constantes en cada paciente los siguientes parámetros de su pau-

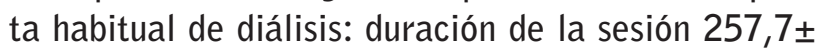
20,3 , flujo de sangre $355,9 \pm 38,3$, calibre de agujas $15 \mathrm{G}$ el $89,5 \%$ y $16 \mathrm{G}$ el $10,5 \%$. Se mantuvo a cada paciente el tipo de heparina usual y las dosis normalmente utilizadas. El flujo de líquido de diálisis fue de $750 \mathrm{ml} / \mathrm{min}$ en HDF y HD-AF y de $500 \mathrm{ml} / \mathrm{min}$ en AFB-K, pues esta técnica no permite que sea más levado. El volumen de perfusión real total administrado en cada sesión fue de $8.677 \pm 430 \mathrm{ml}$ en HDF y $8.316,02 \pm 1.016,75 \mathrm{ml}$ en AFB-K.

A los 15 días de comenzar cada periodo se le realizaba a cada paciente en la sesión de la mitad de la semana un electrocardiograma y una analítica pre y post-diálisis. La analítica incluía: bicarbonato, urea, sodio, potasio, hematocrito y hemoglobina; los datos del $\mathrm{K} / \mathrm{tV}$ se extrajeron de la analítica mensual rutinaria que se realiza durante la primera semana de cada mes.

Se confeccionó una hoja de recogida de datos en la que se anotaba en cada sesión: dializador, peso seco, peso inicial, peso final, ganancia interdiálisis, ultrafiltarción, calibre de las agujas, duración de la sesión, flujos de sangre y líquido de diálisis, presión venosa media, presión arterial media, litros de perfusión prescritos, litros de perfusión administrados, litros de sangre depurados, dosis de eritropoyetina o darbepoetina administrada, dosis de heparina, tensión arterial y frecuencia cardiaca pre y post-diálisis, estado de coagulación del circuito y del dializador, sintomatología intradiálisis (hipotensiones, calambres, náuseas, vómitos, mareos, cefaleas, prurito), especificando el minuto de la sesión en que se producía y la cantidad de suero fisiológico o expansores del plasma que se administraba.

Se utilizó un monitor Integra ${ }^{\circledR}$, equipado con filtro para la retención de endotoxinas en el líquido de baño y capaz de realizar los tres tipos de tratamiento; dializadores: polisulfona y de poliariletersulfona siguiendo la pauta habitual de cada paciente.

Para la AFB-K se utilizaron bolsas para perfusión Hospalsol ${ }^{\circledR}$, conteniendo $166 \mathrm{mEq} / \mathrm{l}$ de bicarbonato y bolsas de concentrado Saferbag $(\AA$ con dos compartimentos, conteniendo el potasio en uno solo de ellos. Para la HDF se utilizaron bolsas para perfusión de HDF conteniendo $60 \mathrm{mEq} / \mathrm{l}$ de bicarbonato y para todas las técnicas concentrado ácido y cartucho de bicarbonato en polvo.

Para el análisis de los datos obtenidos se ha utilizado el programa R-SIGMA. Los datos comparativos entre los distintos tipos de diálisis se analizaron mediante ANOVA para un factor y cuando aparecían diferencias significativas empleábamos la técnica de Neuman-Keuls.

\section{Resultados}

La tensión diastólica posthemodiálisis es la que muestra mayores diferencias entre los tres tratamientos estudiados, obteniendo valores superiores con el método AFB-K. Las frecuencias cardíacas presentan diferencias significativas en los valores posthemodiálisis siendo los más elevados en la hemodiálisis de alto flujo (figura 1).

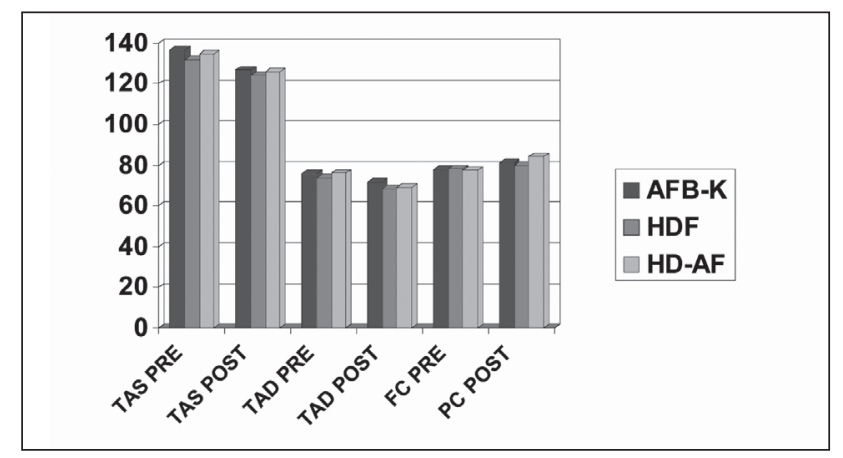

Figura 1. Valores pre y post hemodiálisis de la tensión arterial con las tres técnicas 
En referencia a los diferentes pesos, ganancias interdiálisis y ultrafiltraciones realizadas observamos que no existen diferencias significativas entre un método u otro (tabla 1 ).

\begin{tabular}{|l|c|c|c|c|}
\hline & AFB-K & HDF & HD-AF & \\
\hline Peso pre & $72.55 \pm 15.98$ & $71.48 \pm 14.19$ & $71.27 \pm 15.07$ & NS \\
\hline Peso post & $70.95 \pm 15.72$ & $69.61 \pm 14.24$ & $69.26 \pm 14.88$ & NS \\
\hline Ganancia de peso & $2.34 \pm 1.31$ & $2.26 \pm 1.25$ & $2.30 \pm 1.11$ & NS \\
\hline Ultrafiltración & $2.56 \pm 0.87$ & $2.53 \pm 0.97$ & $2.57 \pm 0.82$ & NS \\
\hline Sangre depurada & $92.38 \pm 11.40$ & $89.61 \pm 12.82$ & $91.96 \pm 14.63$ & NS \\
\hline
\end{tabular}

Tabla 1. Relación de peso, ultrafiltración y sangre depurada en cada técnica en la HD-AF. Los datos revelan un mayor número de calambres en la AFB-K. Mientras que los mareos aparecen más con el método HDF. En cuanto a los registros electrocardiográficos vemos que tanto en la AFB-k como en la HD-.AF los valores de arritmias pre y post no se han modificado pero en la HDF han aumentado razonablemente, siendo esta técnica en la que se registraron mayor número de alteraciones (figura 2).
En las tres técnicas aplicadas encontramos una diferencia sustancial en el valor del bicarbonato pre y post diálisis: En la técnica AFB-K y en la HD-AF el valor del bicarbonato es más fisiológico mientras que con el método HDF ambos valores pre y post son más elevados. En cuanto al sodio pre y post hemodiálisis existen pequeñas diferencias en las tres técnicas en cuanto a los valores totales, estando dentro del intervalo fisiológico. Por último, en referencia a los valores iónicos comentar que en los tres métodos hay una disminución pronunciada de los niveles de potasio, obteniendo el resultado más bajo con la AFB-K, señalar que los valores de $\mathrm{K} / \mathrm{tV}$ son similares en todas las técnicas (tabla 2)

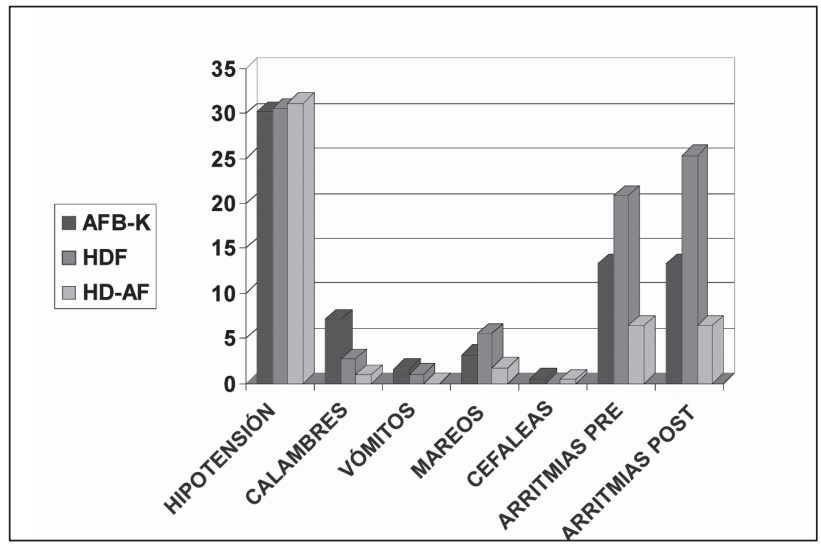

Figura 2. Efectos indeseables durante el tratamiento con las tres técnicas

\begin{tabular}{|l|c|c|c|c|}
\hline & AFB-K & HDF & HD-AF & \\
\hline K/tV & $1.66 \pm 0.29$ & $1.70 \pm 0.31$ & $1.68 \pm 0.30$ & NS \\
\hline Ph pre & $7.34 \pm 0.07$ & $7.41 \pm 0.06^{\mathrm{a}}$ & $7.34 \pm 0.07$ & $\mathrm{p}<0.01$ \\
\hline Ph post & $7.44 \pm 0.06$ & $7.47 \pm 0.08^{\mathrm{a}}$ & $7.44 \pm 0.05$ & $\mathrm{p}<0.01$ \\
\hline Bicarbonato pre & $22.48 \pm 3.85$ & $27.85 \pm 3.63^{\mathrm{a}}$ & $23.43 \pm 3.64^{\mathrm{b}}$ & $\mathrm{p}<0.01$ \\
\hline Bicarbonato post & $28.06 \pm 1.96$ & $33.09 \pm 2.29^{\mathrm{a}}$ & $28.63 \pm 1.89^{\mathrm{b}}$ & $\mathrm{p}<0.01$ \\
\hline Sodio pre & $138.75 \pm 3.51^{\mathrm{c}}$ & $139.23 \pm 3.53^{\mathrm{a}}$ & $137.66 \pm 4.17$ & $\mathrm{p}<0.01$ \\
\hline Sodio post & $136.44 \pm 3.05$ & $138.66 \pm 2.11^{\mathrm{a}}$ & $137.87 \pm 2.78 \mathrm{~b}$ & $\mathrm{p}<0.01$ \\
\hline Potasio pre & $5.06 \pm 0.70^{\mathrm{d}}$ & $4.47 \pm 0.86$ & $5.32 \pm 0.80^{\mathrm{e}}$ & $\mathrm{p}<0.01$ \\
\hline Potasio post & $2.86 \pm 0.28$ & $2.92 \pm 0.38^{\mathrm{b}}$ & $3.30 \pm 0.41^{\mathrm{e}}$ & $\mathrm{P}<0.01$ \\
\hline
\end{tabular}

${ }^{a} p<0.01$ vs AFB-K y HD-AF ${ }^{b} p<0.01$ vs AFB-K ${ }^{c} p<0.01$ vs HD-AF ${ }^{d} p<0.01$ vs HDF ep $<0.01$ vs AFB-K y HDF

Tabla 2. Valores analíticos con las tres técnicas

\section{Discusión}

Diversos estudios contrastados demuestran que con la técnica de AFB-K disminuyen los episodios de arritmias cardiacas ${ }^{6-8}$ aunque no lo hemos constatado en nuestros resultados. Creemos que ello puede ser debido a que hemos utilizado para su valoración un electrocardiograma pre y post diálisis y quizá hubiese sido más adecuado utilizar métodos continuos de registro cardiaco.

En cuanto a la aparición de efectos indeseables durante la diálisis, la diferencia en la aparición de hipotensiones según la técnica utilizada es pequeña, aunque observamos que aparecen menos hipotensiones cuando se utiliza la AFB-K y éstas aumentan
El sistema de AFB-K está ideado, entre otras cosas, para una mejor regulación del equilibrio ácido-bási$\mathrm{co}^{3,6}$. Nuestros resultados así lo corroboran. Observamos un aumento de los niveles de bicarbonato pre y post en la HDF, lo cual nos ha hecho reflexionar sobre 
la idoneidad de la concentración de bicarbonato de las bolsas de líquido de reposición que utilizamos en este método. De hecho, una vez finalizado el estudio, se decidió cambiar las bolsas de $60 \mathrm{mEq} / \mathrm{l}$ por otras que contienen $40 \mathrm{mEq} / \mathrm{l}$.

Como era de esperar la eficacia de las técnicas fue similar en cuanto a $\mathrm{K} / \mathrm{tV}$, y aunque no hemos incluido en nuestro estudio la depuración de medianas moléculas hay múltiples referencias bibliográficas que confirman la superioridad de AFB-K y HDF con respecto a HD-AF en este aspecto $0^{2,4,7}$.

Es de señalar el aumento del número de calambres con AFB-K, lo cual puede estar relacionado con unos valores más bajos de sodio pre y post en dicha técnica. No se puede afirmar que ninguna de las tres técnicas sea superior a las demás y generalizable para todos los enfermos. Lo que es realmente importante es analizar detenidamente las características individuales de cada paciente y optar por la técnica más apropiada para cada caso concreto creemos que mientras la HD-AF sería una opción perfectamente válida en pacientes jóvenes con posibilidad de trasplante y que lleven poco tiempo en programa de diálisis; la HDF sería apropiada para pacientes que llevan mucho tiempo en programa por su mayor capacidad para depurar medianas moléculas y así prevenir complicaciones a largo plazo (amiloidosis, síndrome del túnel carpiano...); mientras que la AFB-K estaría especialmente indicada en pacientes con problemas cardiacos, debido ausencia de iones de acetato y a la posibilidad de realizar el perfil de potasio $0^{6-8}$.

\section{Conclusiones}

Concluimos que los tres métodos han demostrado una correcta eficacia dialítica, tolerancia y estabilidad cardiovascular aunque con AFB-K y HD-AF se consigue una mejor corrección del equilibrio ácido-básico. Además, la AFB-K presenta la ventaja de que el flujo de infusión de bicarbonato es regulable, pudiendo con ello controlar la acidosis de forma personalizada, cosa que no ocurre con la HD-AF.
A pesar de esto creemos que hacen falta estudios electrocardiográficos más completos para poder valorar adecuadamente los efectos beneficiosos de la AFB-K en cuanto a la reducción de las arritmias.

\section{Bibliografía}

1. Lerma $D$ et al. Técnicas alternativas para la depuración extracorpórea. En: Nefrología clínica (formación continuada LOGOSS). 2007: 282-300.

2. Martín de Francisco AL et al. Hemofiltración y Hemodiafiltración. En: Tratado de Hemodiálisis. $2^{a}$ ed. Barcelona: Médica Jims; 2006:271-85.

3. Menica I et al. Valoración de una nueva técnica dialítica: "AFB-K". En: Libro de comunicaciones del XXXI Congreso de la SEDEN. Córdoba 1-4 octubre 2006. Barcelona: HOSPAL; 2006:28386.

4. De la Vara JF: Estudio comparativo de dos modos de tratamiento convectivo: PHF sin acetato, PHF con acetato vs. Hemodiálisis convencional. En: Libro de comunicaciones del XXXI Congreso de la SEDEN. Córdoba 1-4 octubre 2006. Barcelona: HOSPAL; 2006:174-81.

5. López JM, Jofré R y Verde E. Complicaciones cardiacas en el enfermo en hemodiálisis. En: Tratado de Hemodiálisis. $2^{a}$ ed. Barcelona. Médica Jims; 2006:467-81.

6. HOSPAL: El perfil personalizado de potasio: el valor de una mejor respuesta cardiaca. Noviembre 2004.

7. Movilli E et al. A prospective comparison of bicarbonate dialysis, hemodiafiltration, and acetate-free biofiltration in the elderly. Am J Kidney Dis. 1996; 541-7.

8. Santoro A et al: Electrophysiological response to dialysis: the role of dialysate potassium content and profiling. Contrib Nephrol. 2005; 149:295305. 